\title{
EQUILÍBRIO LÍQUIDO-VAPOR A ALTAS PRESSÕES DO SISTEMA DIÓXIDO DE CARBONO + ANETOL: DADOS EXPERIMENTAIS E MODELAGEM MATEMÁTICA
}

\author{
R. SCOPEL SILVA ${ }^{1}$, R. N. ALMEIDA ${ }^{1}$, R. M. F. VARGAS ${ }^{1}$ e E. CASSEL \\ ${ }^{1}$ Pontifícia Universidade Católica do Rio Grande do Sul, Faculdade de Engenharia \\ E-mail para contato: rvargas@ pucrs.br
}

\begin{abstract}
RESUMO - Este trabalho teve como objetivo estudar o equilíbrio líquido-vapor (ELV) a alta pressão do sistema dióxido de carbono + anetol, componente majoritário do óleo essencial de Illicium verum. Foi realizado o levantamento experimental dos pontos de bolha em célula de equilíbrio de volume variável para quatro temperaturas: 298, 303, 308 e $313 \mathrm{~K}$. Com os dados experimentais obtidos foi realizada a modelagem matemática do ELV com auxílio de rotinas de programação implementadas no software Matlab, utilizando as regras de mistura de vdW1 e vdW2 aplicadas à equação de estado de Peng-Robinson. A regra de mistura que melhor representou os dados experimentais foi vW2, com um $\% \Delta \mathrm{P}$ de $6,15 \%$, devido a sua capacidade de levar em conta a assimetria entre as moléculas estudadas. Os parâmetros de interação binária estimados são utilizados no cálculo da solubilidade do oléo essencial de $I$. verum em $\mathrm{CO}_{2}$ ou na predição de sistemas ternários, para posterior modelagem de processos de transferência de massa.
\end{abstract}

\section{INTRODUÇÃO}

O projeto e dimensionamento de processos de separação como destilação, absorção e extração (líquido-líquido, supercrítica) requerem conhecimento detalhado do equilíbrio de fases do sistema multicomponente (Bamberger et al., 2000). O estudo experimental completo, abrangendo todas as condições possíveis de processo é inviável, devido ao custo e ao elevado tempo. O que implica no uso de modelos termodinâmicos capazes de predizer, através de parâmetros ajustados, o equilíbrio de fases do sistema (Jha e Madras, 2005).

A pressões moderadas, os modelos baseados na energia livre de Gibbs em excesso da fase líquida combinados com as expressões de coeficiente de fugacidade para a fase gasosa permitem o cálculo do equilíbrio líquido-vapor (ELV) de forma confiável. Mas esta estratégia falha quando aplicada a sistemas de alta pressão e próximos do ponto crítico. Desta forma, as equações de estado (EOS) são frequentemente utilizadas para descrever o equilíbrio de fases a alta pressão com um de seus componentes na região próxima à crítica (Sieder e Maurer, 2004).

A determinação do equilíbrio de fases para sistemas com fluidos supercríticos é necessária devido ao recente desenvolvimento das suas aplicações e ao reduzido número de dados disponíveis (Cheng et al., 2000). Normalmente na modelagem matemática de processos em que fluidos 


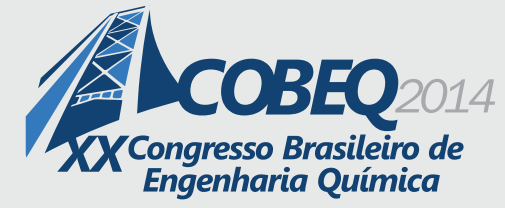

19 a 22 de outubro de 2014

Florianópolis/SC

supercríticos são utilizados como solventes se estimam parâmetros que representam a solubilidade dos compostos orgânicos no solvente a partir dos dados de rendimento versus tempo ou através de equações empíricas em vez de calcular a mesma a partir do uso de equações de estado. Portanto, este trabalho visa obter experimentalmente dados de equilíbrio da mistura binária anetol $+\mathrm{CO}_{2} \mathrm{em}$ alta pressão, para que através de rotinas de programação, seja realizada a modelagem termodinâmica do sistema com a equação de estado Peng Robinson utilizando as regras de mistura van de Waals 1 (vW1) e van der Waals 2 (vW2) Como os óleos essenciais das espécies Illicium verum (anisestrelado) e Pimpinella anisum L. (erva-doce) apresentam em sua composição química aproximadamente $86 \%$ de anetol, justifica-se assim o presente estudo do equilíbrio do sistema $\mathrm{CO}_{2}+$ anetol.

\section{METODOLOGIA}

\subsection{Determinação dos dados experimentais de equilíbrio líquido-vapor}

Os pontos experimentais foram obtidos no equipamento de determinação de equilíbrio em altas pressões do Laboratório de Operações Unitárias (LOPE) apresentado na Figura 1 (Scopel, 2013). O padrão do anetol foi adquirido junto a Sigma-Aldrich, e o dióxido de carbono junto a Air Products $(99,9 \%$ pureza).

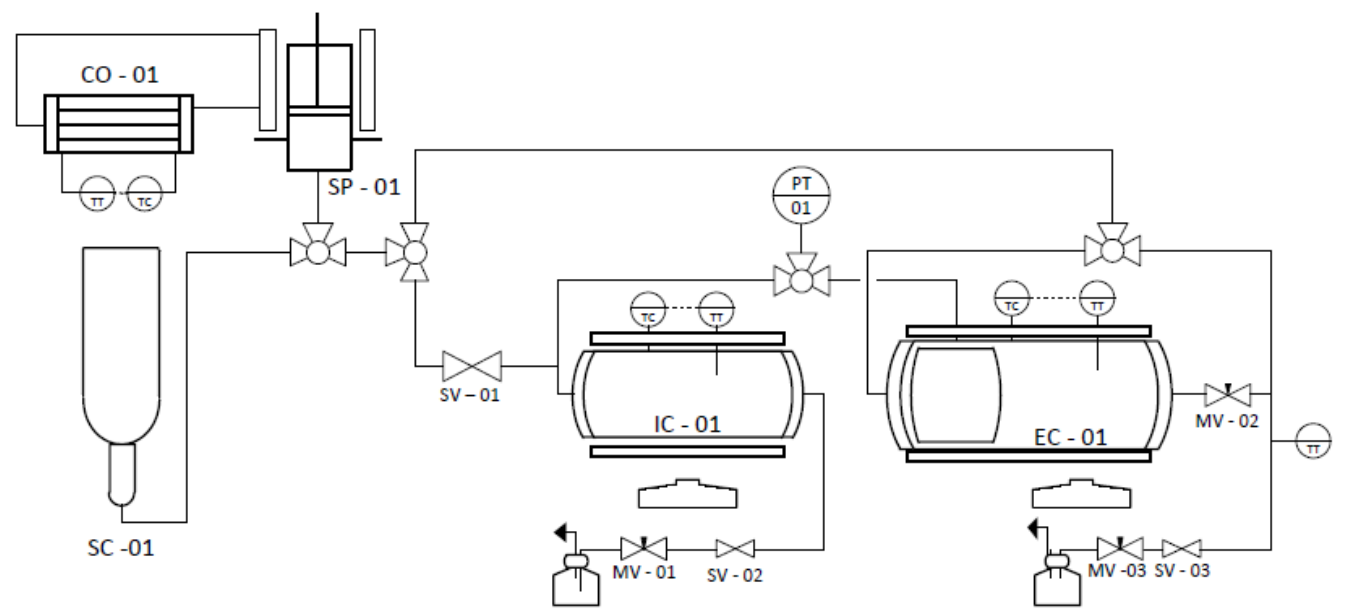

Figura 1 - Fluxograma do equipamento de determinação de equilíbrio de fases em alta pressão.

$\mathrm{O}$ equipamento consiste em uma alimentação de $\mathrm{CO}_{2}$ a uma bomba $(\mathrm{SP}-01)$ com temperatura controlada por um banho termostático. O fluxo é direcionado para a câmara frontal da célula de equilíbrio (EC - 01) através das válvulas. Uma quantidade pré-definida de $\mathrm{CO}_{2}$, levando-se em conta o volume fixo da célula e a fração molar desejada, é injetada na parte da frente da câmara que já possui o composto a ser estudado, neste caso o anetol. A massa de anetol foi determinada em balança de precisão 0,0001g (Marte AW 220). 
A alimentação na parte frontal da célula se dá na pressão de $1000 \mathrm{kPa}$ e $288 \mathrm{~K}$, possibilitando o total conhecimento das propriedades do fluido. Para cada temperatura de interesse, são realizados 08 medidas, variando as pressões, para que seja obtida a tendência dos pontos da curva. A partir de cada experimento, com uma fração molar constante, diferentes pontos de pressão e temperatura no equilíbrio são obtidos.

\subsection{Modelagem do ELV}

Neste trabalho foi utilizada a equação de estado Peng Robinson (Equação 1) para correlacionar o equilíbrio líquido-vapor (ELV) a alta pressão. O critério de equilíbrio utilizado no trabalho foi o da isofugacidade, conforme a Equação 2. De posse dos dados experimentais o tratamento matemático é conduzido através do método simplex Nelder-Mead (Lagarias et al., 1998), implementado no software de programação MATLAB.

$$
\begin{gathered}
P=\frac{R T}{V-b}-\frac{a}{V^{2}+2 b V-b^{2}} \\
\hat{f}_{i}^{v}\left(T, P, y_{i}\right)=\hat{f}_{i}^{l}\left(T, P, x_{i}\right), \quad i=(1,2, \ldots, N)
\end{gathered}
$$

Que por definição resulta em:

$$
\hat{\phi}_{i}^{v} y_{i}=\hat{\phi}_{i}^{l} x_{i}
$$

Na Equação 3, $\hat{\phi}_{i}^{l}$ e $\hat{\phi}_{i}^{v}$ são os coeficientes de fugacidade em mistura do componente $i$ na fase líquida e vapor, respectivamente, e $x_{i}$ e $y_{i}$ as frações molares do componente $i$ também nas fases líquida e vapor.

\subsubsection{Regras de mistura}

A regra de mistura empírica van der Waals 1 (vdW1) utilizada no estudo (Equações 4 e 5) é considerada o modelo clássico,

$$
\begin{gathered}
a=\sum_{i} \sum_{j} z_{i} z_{j} \sqrt{a_{i} a_{j}}\left(1-k_{i j}\right) \\
b=\sum_{i} z_{i} b_{i}
\end{gathered}
$$

onde $\mathrm{k}_{\mathrm{ij}}=\mathrm{k}_{\mathrm{ji}}$ e se $\mathrm{i}=\mathrm{j}, \mathrm{k}_{\mathrm{ij}}=0$. E $\mathrm{z}$ a fração molar na fase analisada.

A segunda regra de mistura utilizada neste estudo, regra de mistura van der Waals 2 (vdw2), e apresentada por Shibata (1989) (Equações 6 e 7) é baseada na regra clássica de van der Waals, mas adiciona um termo binário no equacionamento de $b$. 


$$
\begin{aligned}
a & =\sum_{i} \sum_{j} z_{i} z_{j} \sqrt{a_{i} a_{j}}\left(1-k_{i j}\right) \\
b & =\sum_{i} \sum_{j} z_{i} z_{j} \frac{1}{2}\left(b_{i}-b_{j}\right)\left(1-n_{i j}\right)
\end{aligned}
$$

onde $\mathrm{k}_{\mathrm{ij}}=\mathrm{k}_{\mathrm{ji}}, \mathrm{n}_{\mathrm{ij}}=\mathrm{n}_{\mathrm{ji}}$ e se $\mathrm{i}=\mathrm{j}, \mathrm{k}_{\mathrm{ij}}=0$ e $\mathrm{n}_{\mathrm{ij}}=0$. E $\mathrm{z}$ a fração molar na fase analisada.

Para que a modelagem do ELV seja determinada de maneira correta é necessário garantir o efeito de temperatura no parâmetro atrativo da EOS. A primeira modificação deste tipo foi feita por Soave (1972) na equação de Redlich-Kwong, adicionando a função $\alpha(T)$, determinada a partir do fator acêntrico do composto puro. Segundo Valderrama (2003) um desvio significativo entre os dados experimentais e os dados gerados pelo modelo baseado na EOS são encontrados quando as temperaturas são inferiores a $0,7 T_{c}$. Desta forma, um imenso número de estudos em que parâmetros ajustáveis são introduzidos no equacionamento de $\alpha(T)$ são encontrados na literatura, a partir de dados de pressão de vapor. Neste trabalho se optou pelo modelo proposto por Almeida et al. (1991) que utiliza a temperatura reduzida como variável $\left(T_{r}=T / T_{c}\right)$ e possui $\mu$, $\xi$ e $\varpi$ como parâmetros ajustáveis (Equação 8).

$$
\alpha=\exp \left(\mu \cdot\left(1-T_{r}\right) \cdot\left|1-T_{r}\right|^{\xi-1}+\varpi \cdot\left(\frac{1}{T_{r}}-1\right)\right)
$$

\section{RESULTADOS E DISCUSSÕES}

\subsection{Dados experimentais do ELV}

Na Tabela 1 são apresentados os dados experimentais de ponto de bolha da mistura $\mathrm{CO}_{2}(1) /$ anetol(2) para 04 temperaturas: 298,$15 ; 303,15 ; 308,15 ; 313,15 \mathrm{~K}$.

Tabela 1 - Dados experimentais do ELV CO $2(1) /$ anetol(2) a 298,15; 303,15; 308,15;313,15K

\begin{tabular}{cccccccc}
\hline \multicolumn{2}{c}{$298,15 \mathrm{~K}$} & \multicolumn{2}{c}{$303,15 \mathrm{~K}$} & \multicolumn{2}{c}{$308,15 \mathrm{~K}$} & \multicolumn{2}{c}{$313,15 \mathrm{~K}$} \\
\hline $\mathrm{P}(\mathrm{kPa})$ & $\mathrm{x}_{1}$ & $\mathrm{P}(\mathrm{kPa})$ & $\mathrm{x}_{1}$ & $\mathrm{P}(\mathrm{kPa})$ & $\mathrm{x}_{1}$ & $\mathrm{P}(\mathrm{kPa})$ & $\mathrm{x}_{1}$ \\
\hline 1067,29 & 0,102 & 1124,26 & 0,102 & 1245,32 & 0,102 & 1388,76 & 0,102 \\
1988,97 & 0,200 & 2312,47 & 0,200 & 2868,94 & 0,200 & 3352,16 & 0,200 \\
3249,42 & 0,301 & 3588,17 & 0,301 & 3900,49 & 0,301 & 4274,86 & 0,301 \\
4281,98 & 0,400 & 4738,43 & 0,400 & 5167,04 & 0,400 & 5637,03 & 0,400 \\
5252,49 & 0,503 & 5724,52 & 0,503 & 6321,68 & 0,503 & 6937,15 & 0,503 \\
5495,63 & 0,598 & 6077,52 & 0,598 & 6716,39 & 0,598 & 7429,53 & 0,598 \\
6140,60 & 0,700 & 6663,49 & 0,700 & 7458,01 & 0,700 & 8306,44 & 0,700 \\
6205,00 & 0,802 & 6745,89 & 0,802 & 7616,71 & 0,802 & 8627,91 & 0,802 \\
\hline
\end{tabular}




\subsection{Modelagem do ELV}

Conforme mencionado anteriormente, foi utilizada a EOS-PR com duas diferentes regras de mistura para a representação do ELV do sistema $\mathrm{CO}_{2}(1) /$ anetol(2). Ao realizar a modelagem se observa que quanto mais próximo ao ponto crítico, o sistema se torna mais instável, principalmente para a regra de mistura vdW1.

Conforme foram realizados os cálculos foi identificado que para os pontos extremos, ou seja, os mais próximos do ponto crítico, os modelos não convergem por se tratar de uma mistura crítica e não um equilíbrio líquido-vapor. Portanto são desconsiderados os pontos próximos ao ponto crítico para efeitos de cálculo. Os parâmetros de interação binária gerados são apresentados nas Tabelas 2 e 3 , respectivamente para as regras de mistura vdW1 e vdW2. O erro $(\% \Delta \mathrm{P})$ máximo para a regra vdW1 é de $18,56 \%$ e média de $15,05 \%$. Para a regra de mistura vdW2 o erro máximo é de $6,15 \%$ e média de 5,38\%. Portanto, o último modelo mencionado é o que melhor se ajusta aos dados experimentais.

Tabela 2 - Parâmetros binários da EOS-PRvdW1 estimados para a mistura $\mathrm{CO}_{2}(1) / \operatorname{anetol}(2)$

\begin{tabular}{ccccc}
\hline \multicolumn{5}{c}{$\mathbf{v d W 1}$} \\
\hline $\mathbf{T}(\mathbf{K})$ & 298,15 & 303,15 & 308,15 & 313,15 \\
$\mathbf{k}_{\mathbf{1 2}}$ & 0,0955 & 0,0921 & 0,0916 & 0,0916 \\
$\mathbf{\%} \mathbf{\Delta}$ & 11,01 & 14,01 & 16,61 & 18,56 \\
\hline
\end{tabular}

Tabela 3 - Parâmetros binários da EOS-PRvdW2 estimados para a mistura $\mathrm{CO}_{2}(1) /$ anetol(2)

\begin{tabular}{ccccc}
\hline \multicolumn{5}{c}{ vdW2 } \\
\hline $\mathbf{T}(\mathbf{K})$ & 298,15 & 303,15 & 308,15 & 313,15 \\
$\mathbf{k}_{\mathbf{1 2}}$ & 0,0843 & 0,0790 & 0,0738 & 0,0695 \\
$\mathbf{n}_{\mathbf{1 2}}$ & $-0,0190$ & $-0,0235$ & $-0,0306$ & $-0,0367$ \\
$\mathbf{\%} \mathbf{\Delta}$ & 4,49 & 4,96 & 5,92 & 6,15 \\
\hline
\end{tabular}

Os gráficos dos pontos de bolha experimentais gerados pelas equações de estados PRvdW1 e PRvdW2 para a mistura binária $\mathrm{CO}_{2}(1) / \operatorname{anetol}(2)$, nas quatro temperaturas estudadas, podem ser visualizados na Figura 2. É possível observar que as curvas geradas pela EOS-PRvdw2 são as que melhores se ajustam aos pontos experimentais. Observa-se também que os desvios entre os dados experimentais e os dados calculados pela EOS-PRvdw1 se acentuam para altas concentrações de $\mathrm{CO}_{2}$, fato esperado previamente em função da definição o termo repulsivo da regra de mistura. 


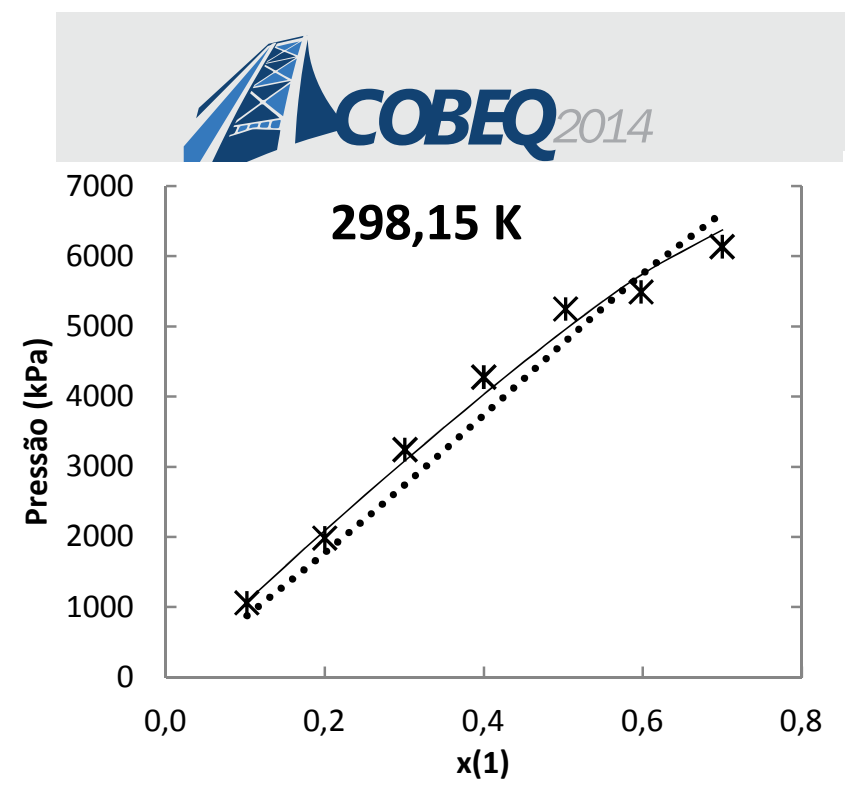

(a)

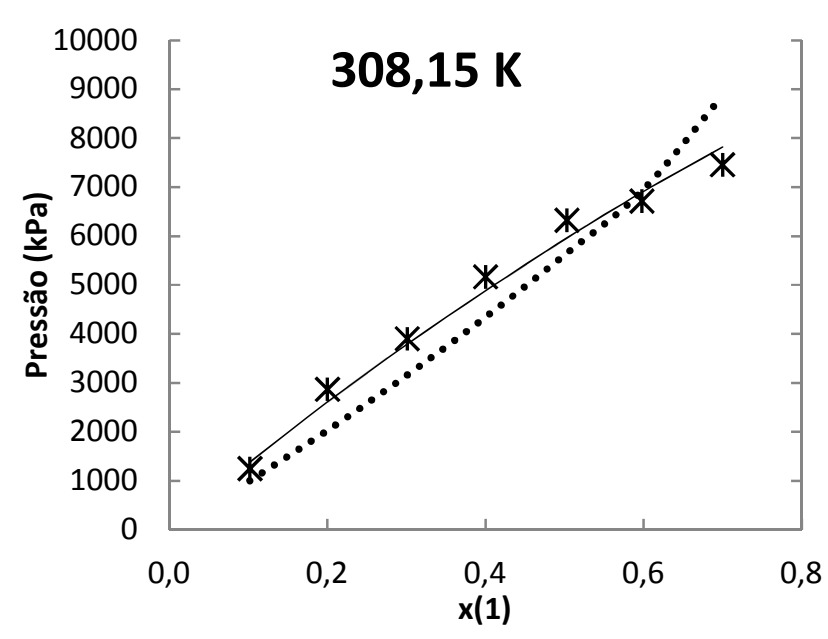

(c)
19 a 22 de outubro de 2014

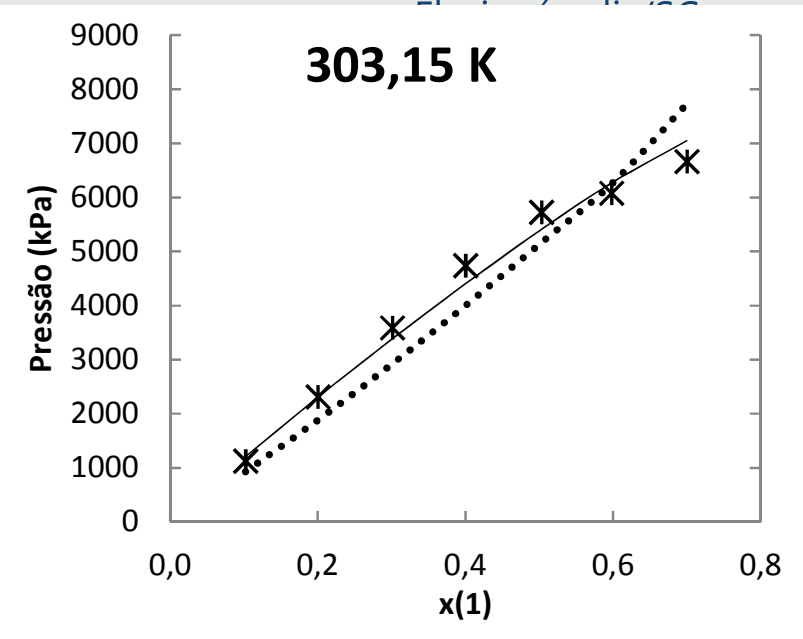

(b)

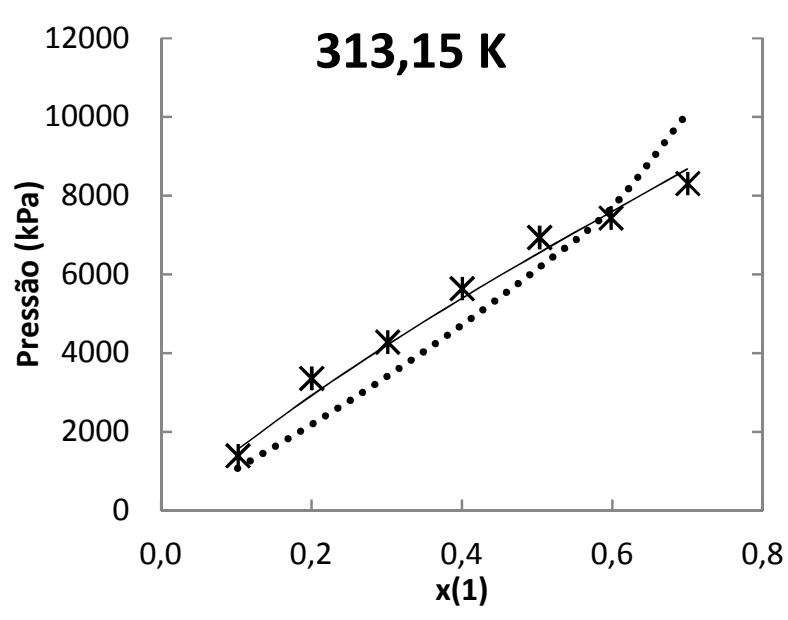

(d)

Figura 2 - Ponto de bolha representado pelos dados de pressão versus fração molar de $\mathrm{CO}_{2}$ da mistura binária $\mathrm{CO}_{2}(1) /$ anetol(2), sendo (ж) dados experimentais, (.....) vdW1 e (一) vdW2 nas temperaturas (a) 298,15 K; (b) 303,15 K; (c) 308,15 K; (d) $313,15 \mathrm{~K}$.

\section{CONCLUSÕES}

Os dados experimentais do ELV do sistema $\mathrm{CO}_{2}+$ anetol nas temperaturas de 298, 303, 308 e $313 \mathrm{~K}$ obtidos nestes estudos subsidiaram a modelagem termodinâmica com a EOS-PR da mistura $\mathrm{CO}_{2}$ (1)/anetol(2), possibilitando assim a avaliação do comportamento das regras de mistura vW1 e vW2 na representação dos dados experimentais da mistura em questão. Conclui-se que leva a regra de mistura $\mathrm{vW} 2$ apresentou o menor erro $(\% \Delta \mathrm{P})$ em relação aos valores experimentais fruto da incorporação do parâmetro ajustável $\mathrm{n}_{\mathrm{ij}}$ no termo $\mathrm{b}$ da regra de mistura. Este parâmetro associa à EOS as assimetrias entre as moléculas estudadas, o que não é considerado no modelo vdW1, sendo feito 


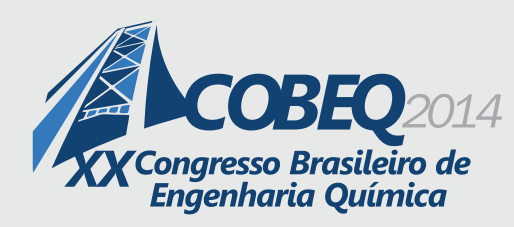

apenas uma ponderação do efeito de cada componente da mistura.

Os parâmetros determinados neste trabalho podem ser utilizados para o cálculo da solubilidade dos óleos essenciais das espécies Illicium verum (anis-estrelado) e Pimpinella anisum L. (erva-doce) com $\mathrm{CO}_{2}$, visto que o anetol é o composto majoritário nestes extratos voláteis, alcançando valores médios de $86 \%$ da composição total.

\section{REFERÊNCIAS}

ALMEIDA, G. S., AZNAR, M., TELlES, A. S., Uma Nova Forma de Dependência com a Temperatura do Termo Atrativo de Equações de Estado Cúbicas. RBE - Cad. Eng. Quim, v. 8, p. 95, 1991.

BAMBERGER, A., SIEDER, G., MAURER, G. High-pressure (vapor+ liquid) equilibrium in binary mixtures of (carbon dioxide+ water or acetic acid) at temperatures from 313 to $353 \mathrm{~K}$. J Supercrit Fluid, v. 17, p. 97-110, 2000.

CHENG, K. W., KUO, S. J., TANG, M., \& CHEN, Y. P. Vapor-liquid equilibria at elevated pressures of binary mixtures of carbon dioxide with methyl salicylate, eugenol, and diethyl phthalate. J Supercrit Fluid, v. 18, p. 87-99, 2000.

JHA, S. K., MADRAS, G. Correlations for binary phase equilibria in high-pressure carbon dioxide. Fluid Phase Equilibr, 238, p.174-179, 2005.

LAGARIAS, J. C. et al. Convergence properties of the Nelder--Mead simplex method in low dimensions. J Optimiz, v. 9, p. 112-147, 1998.

PENG, D-Y., ROBINSON, D. B. A new two-constant equation of state. Ind Eng Chem Fund, v. 15, p. 59-64, 1976.

POLING, B. E., PRAUSNITZ, J. M., JOHN PAUL, O. C., \& REID, R. C.. The properties of gases and liquids. New York: McGraw-Hill, 2001.

SCOPEL, R. S., Incorporação de modelos termodinâmicos na modelagem matemática de processos de extração supercrítica. Dissertação de Mestrado em Engenharia, Faculdade de Engenharia, Pontifícia Universidade Católica do Rio Grande do Sul, Porto Alegre, 2013. 84p.

SHIBATA, S. K., SANDLER, S. I. Critical evaluation of equation of state mixing rules for the prediction of high-pressure phase equilibria. Ind Eng Chem Res, v. 28, p. 1893-1898, 1989.

SIEDER, G., MAURER, G. An extension of the Peng-Robinson equation of state for the correlation and prediction of high-pressure phase equilibrium in systems containing supercritical carbon dioxide and a salt. Fluid Phase Equilibr, v. 225, p. 85-99, 2004.

SMITH, J. M., VAN NESS H. C., ABBOTT, M. M., Introdução à Termodinâmica da Engenharia Química. LTC Ltda, 2007.

SOAVE, G. Equilibrium constants from a modified Redlich-Kwong equation of state. Chem Eng Sci, 
v. 27, p. 1197-1203, 1972.

SOLÓRZANO-ZAVALA, M., BARRAGÁN-AROCHE, F., BAZÚA, E. R. Comparative study of mixing rules for cubic equations of state in the prediction of multicomponent vapor-liquid equilibria. Fluid Phase Equilibr, v. 122, p. 99-116, 1996.

VALDERRAMA, J. O. The state of the cubic equations of state. Ind Eng Chem Res, v. 42, p. 1603$1618,2003$.

VAN DER WAALS, J. D. Over de Continuiteit van den Gas-en Vloeistoftoestand. AW Sijthoff, 1873. 